\title{
Cyclical changes in deformation process in granular material in active state
}

\author{
Magdalena Pietrzak ${ }^{1, *}$ \\ ${ }^{1}$ Koszalin University of Technology, Department of Civil Engineering, Environmental and Geodetic Science, Koszalin, Poland
}

\begin{abstract}
This paper presents a detailed study of a selected small scale model test, performed on a sample of surrogate granular material, retained by a rigid wall (typical geotechnical problem of earth thrust on a retaining wall). The experimental data presented in this paper show that the deformation of granular sample behind retaining wall can undergo some cyclic changes. The nature of these cycles is not clear - it is probably related to some micromechanical features of granular materials, which are recently extensively studied in many research centers in the world. Employing very precise DIC (PIV) method can help to relate micro and macro-scale behavior of granular materials.
\end{abstract}

\section{Introduction}

This paper presents a detailed study of a selected small scale model test, performed on a sample of surrogate granular material, retained by a rigid wall (typical geotechnical problem of earth thrust on a retaining wall). The model wall translated horizontally in an active mode (out of the granular sample).

The reason for presenting the test in such a way is that several unique observations on evolution of failure mechanism were observed within the granular sample, which seem not to be described earlier in literature. The observations were confirmed in a number of similar tests. The new findings were possible to be made due to employing image analysis, namely DIC (PIV) in elaboration of test results.

\section{Model tests}

The experimental set-up applied in this work was described in detail in [1-5]. Small-scale tests on granular samples retained by a movable rigid wall were performed in a glass-sided box (Fig.1). The glass sides were $20 \mathrm{~mm}$ thick and loaded by lateral pressures from the granular material. The particular configuration included a smooth and rigid vertical wall, $180 \mathrm{~mm}$ high, supported by rods that were able to slide horizontally through the box. An active earth pressure mode was investigated, where a retaining wall moved away from the backfill. The mode of the test was quasi static with a constant wall displacement increment equal to $0.0625 \mathrm{~mm}(1 / 20$ of the supporting screw lead).

The tests were recorded by a common digital camera Sony Cyber shot with the resolution of 2560 x 1920 pixels. One average grain was represented by $\sim 10 \mathrm{x} 10$ pixels. Photographs were taken at each wall displacement step. Experimental displacement fields were determined on their base and hence strains calculated, using 2D digital image correlation (particle image velocimetry) DIC (PIV). Image analysis software geoPIV RG, elaborated by White and Take [6 - 8] was employed.

The tests were so-called 'constrained' tests, during which the surface loading was provided by a footing formed of five rigid segments connected to the common pressure source, so that the pressure on each segment could be maintained as the footing articulated and settled (Fig.1). The granular sample was loaded up to $1.6 \mathrm{MPa}$ in eight steps by $0.2 \mathrm{MPa}$. At pressure equal to $1.6 \mathrm{MPa}$, the wall was permitted to move 'actively' (out of the specimen) by releasing the support screw in constant increments.

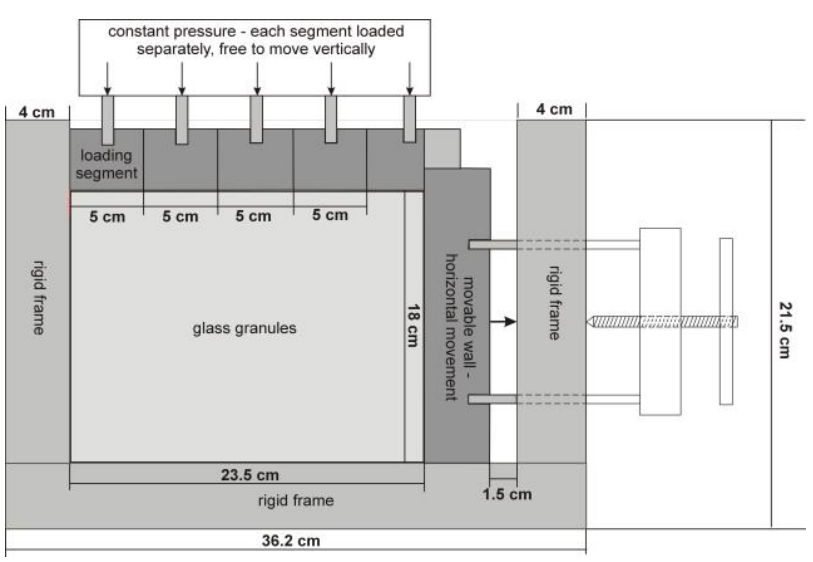

Fig. 1. Experimental setup.

\footnotetext{
* Corresponding author: magdalena.pietrzak@tu.koszalin.pl
} 


\section{Materials}

Starlitbeads1000 spherical glass granules (Fig.2) were used to form a granular sample $(\mathrm{d} 50=1.1 \mathrm{~mm})$. Only dense samples were investigated. To produce a dense sample, granular material was rained into the box from a hopper. The glass granules were selected to represent soil due to their transparency. The tests were registered both in ordinary and polarized light, to give not only strain, but also stress information based on photo-elasticity, but the latter is not a subject of this paper. Due to the demands of photo-elastic method the granular specimen was saturated with clove oil, having the same refraction index as glass [1-4].
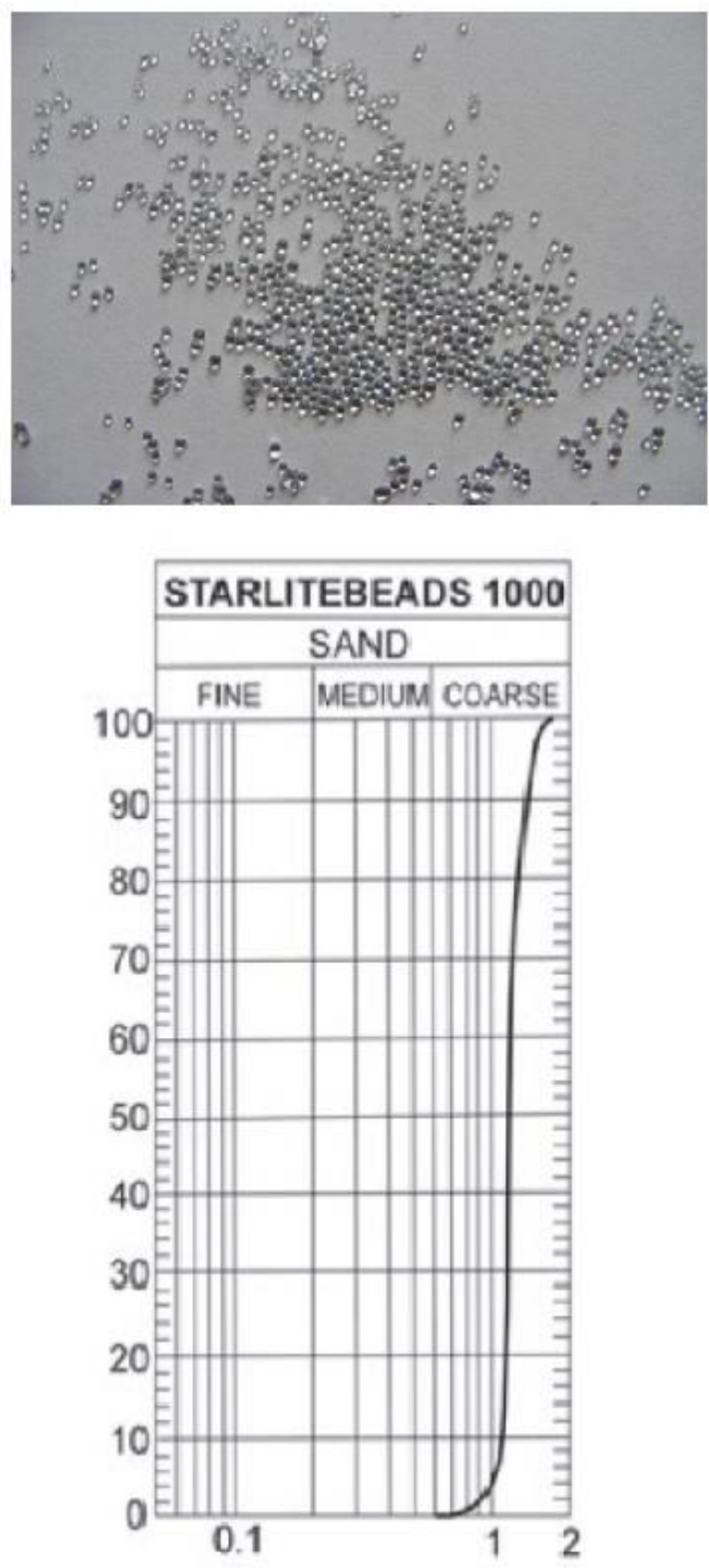

Fig. 2. View on glass particles and sieve test results.

\section{Discussion of the model test results}

The main subject of discussion are deformation fields (shear and volumetric), obtained in experimental model tests by means of image analysis (DIC). Unlike earlier studies, known from the literature, in which the final effect of deformation with large increases in wall displacements (cumulative deformations) was studied, the paper focuses on a thorough investigation of the evolution process of deformation fields, and therefore their analysis was carried out for each step of the experiment. Such a step consisted of either an increase in the external load or an increase in the horizontal displacement of the retaining wall model in a direction 'from the ground' (Fig.1). The basic increase in wall displacement was $\delta \mathrm{d}=0.0625 \mathrm{~mm}$, i.e. it was by an order of magnitude smaller than the average grain diameter of tested granular materials. The ratio of this increase to the wall height was approximately $3.5 \times 10-4$. In all cases, both shear and volumetric strains show some similarities, regardless of the applied load level, but also some characteristic differences. Common features are, for example, the occurrence of horizontal locations of shear and volumetric strains in the straight loading phase of the model and clear shear band cutting off the area of material adjacent to the displacement wall, appearing at certain stages. The alternation of behavior is also clearly visible for all tests carried out using the minimum wall displacement: the moments of a clear shear band are separated by steps in which deformations are distributed, but occur within the shear band of the wedge.

This alternation (cyclicality) of the model behavior is very clearly visible in Test 4 , and therefore it will be used for a more detailed description of the characteristic deformation features. Watching the full course of Test 4 , it can be seen that the wall displacement needed for subsequent episodes of localized shear band was $3-4 \delta \mathrm{d}$, i.e. approximately $0.25 \mathrm{~mm}$ (i.e. approximately $1 / 4$ of the average grain diameter).

From the full course of Test 4 , shear and volumetric strains were selected for detailed discussion, presented in Fig.3-9. They illustrate several characteristic processes observed in the course of practically all experiments. These include: reaction to external load in the phase without wall displacements, the cyclic nature of the strains localization, applied on the scheme of progressive deformation development.

\subsection{Effect of external loading on the deformation field}

In the first phase of Test 4 , the model was gradually loaded to $1.6 \mathrm{MPa}$, using an increment equal to $0.2 \mathrm{MPa}$, with the wall immobilized. As a result of this loading, despite the lack of wall displacements, local diffuse changes in shear and volumetric strains, related to the concentration of granules under load, were observed (Fig.3.). As can be seen in Fig.3a, despite the fact that load was based on single-axis compression from above, local shear strains appeared in the medium under 


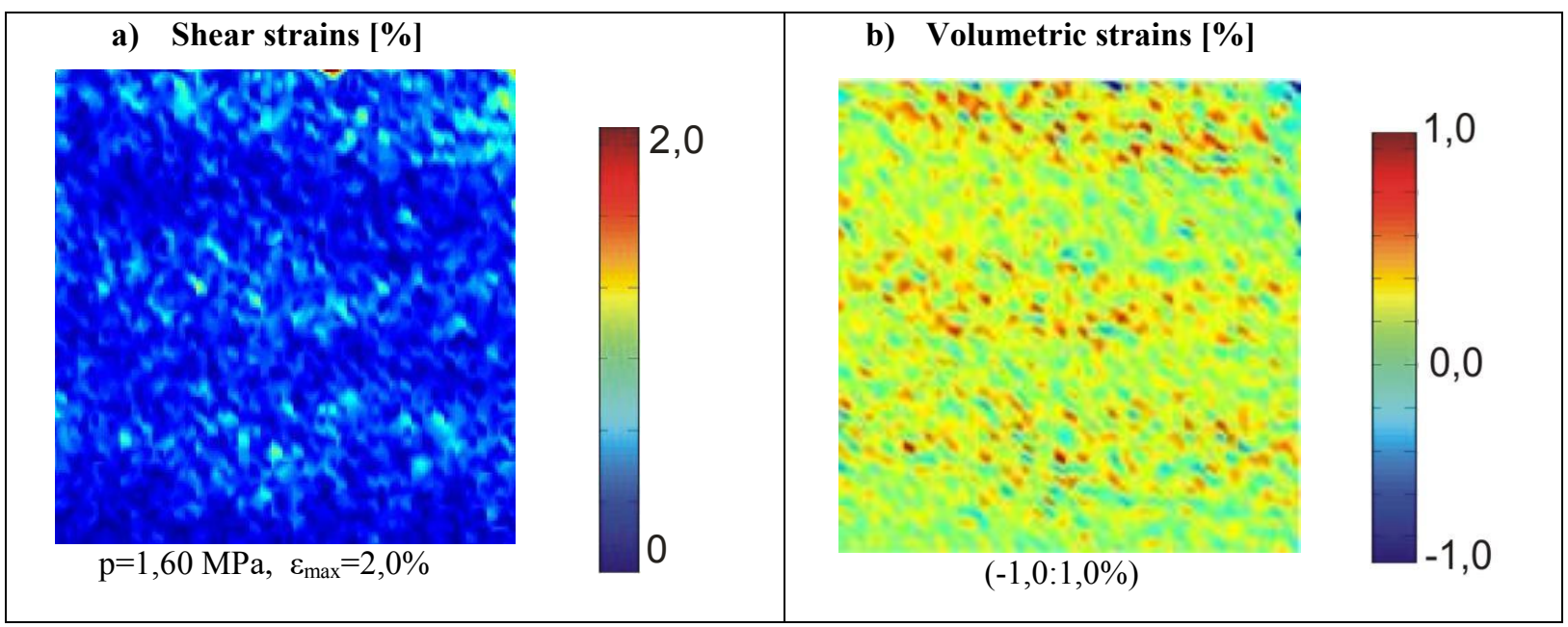

Fig. 3. Initial stage: 'Compaction bands' due to external loading of the granular sample.

\section{a) Shear strains $[\%]$}

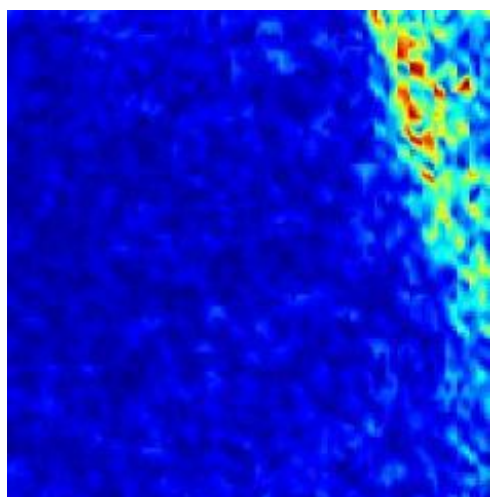

$\varepsilon_{\max }=1,2 \% \mathrm{~d}=0,5000 \mathrm{~mm}$

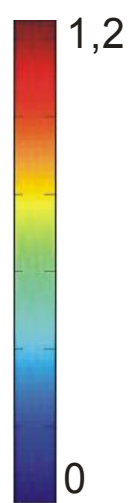

\section{b) Volumetric strains [\%]}

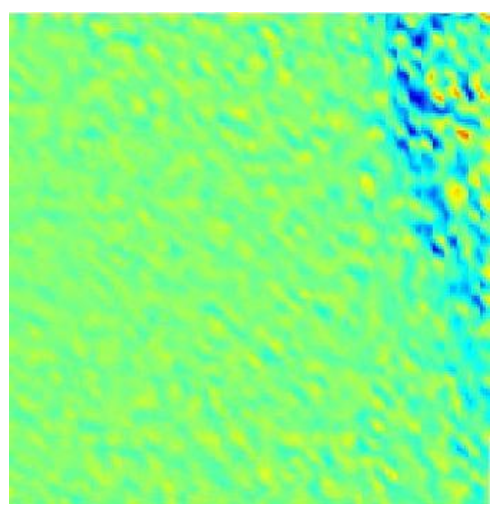

$(-1,0: 1,0 \%)$

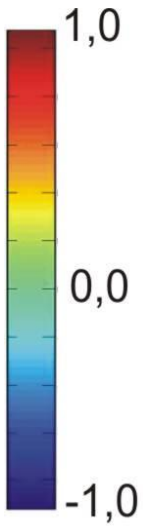

Fig. 4. Stage preceding the localization episode: distributed deformations in the wedge forming area (a). Prevalence of dilating (blue) at the edge of wedge and compression inside it (red) (b).

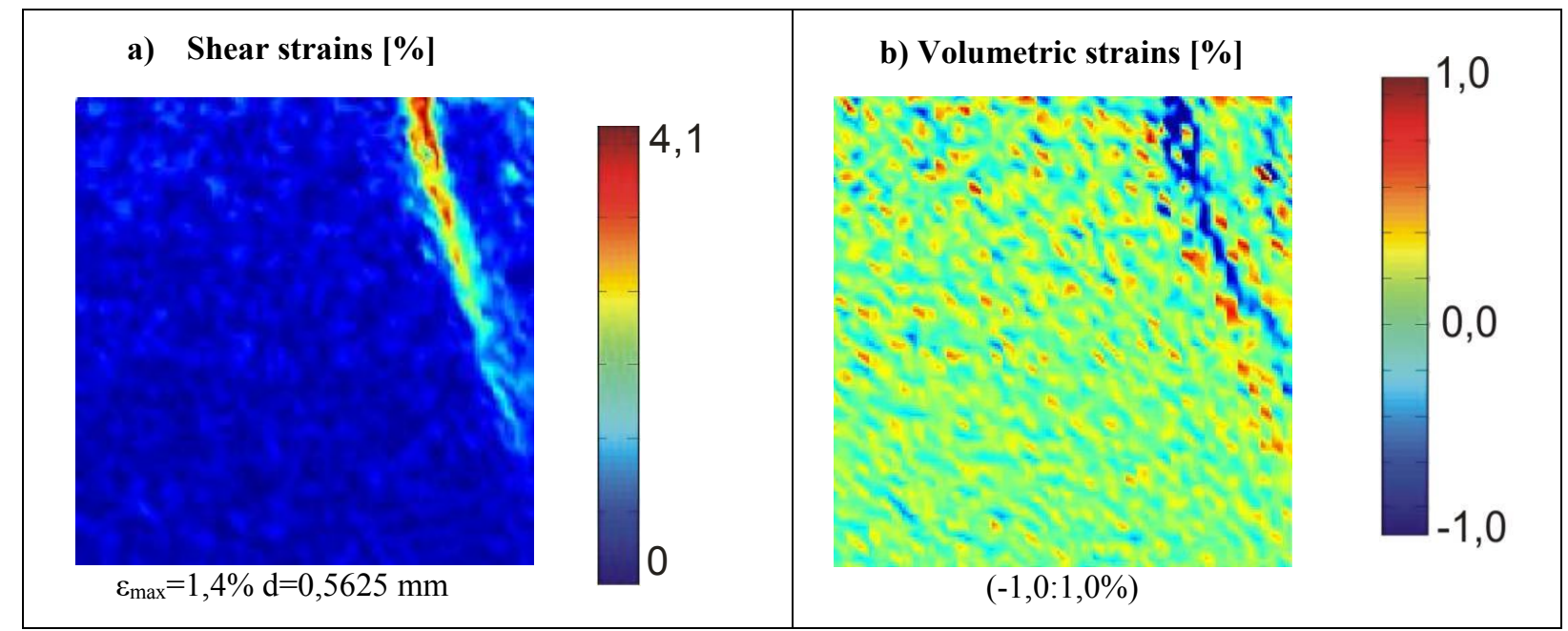

Fig. 5. Localization episode: deformations concentrated inside the shear band (a). Volume increase (b), along the entire length of localization (blue), compression (red) at its edges.

\footnotetext{
*Corresponding author: magdalena.pietrzak@tu.koszalin.pl
} 


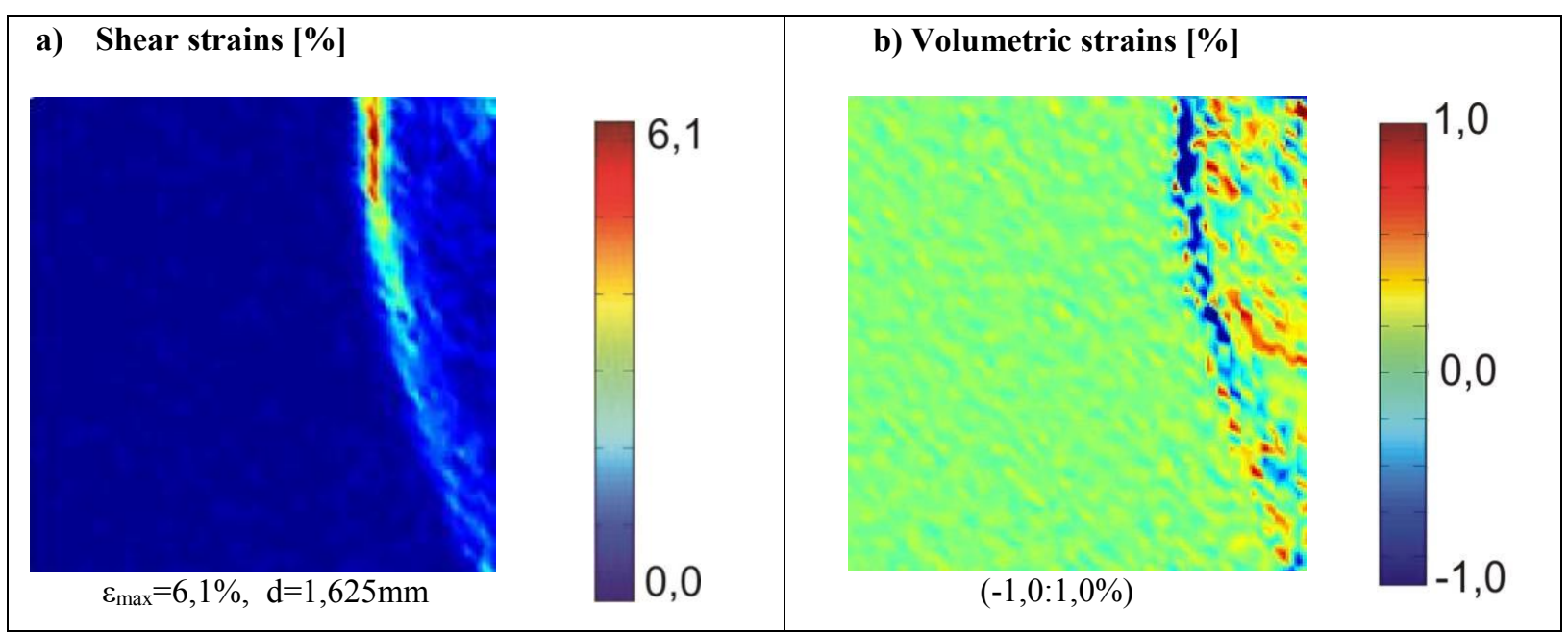

Fig. 6. Localization episode: developing shear band - deformations concentrated inside the shear band (a), their size depends on the position inside the shear band (the largest at the top). (b) Volume increase (blue) along the entire length of localization, compression (yellow and red) inside the wedge.

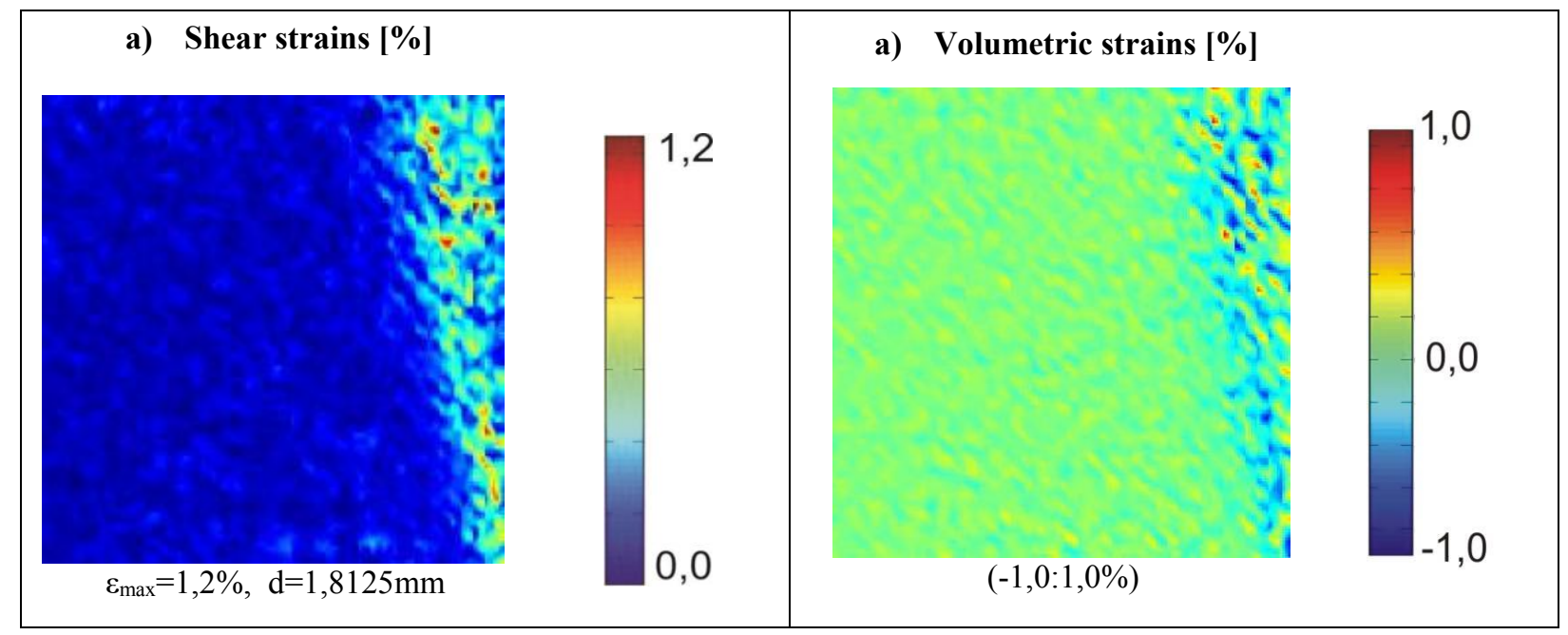

Fig. 7. Stage after localization: distributed deformation in the wedge forming area. (b) Dilatant advantage (volume increase, blue) inside the wedge.

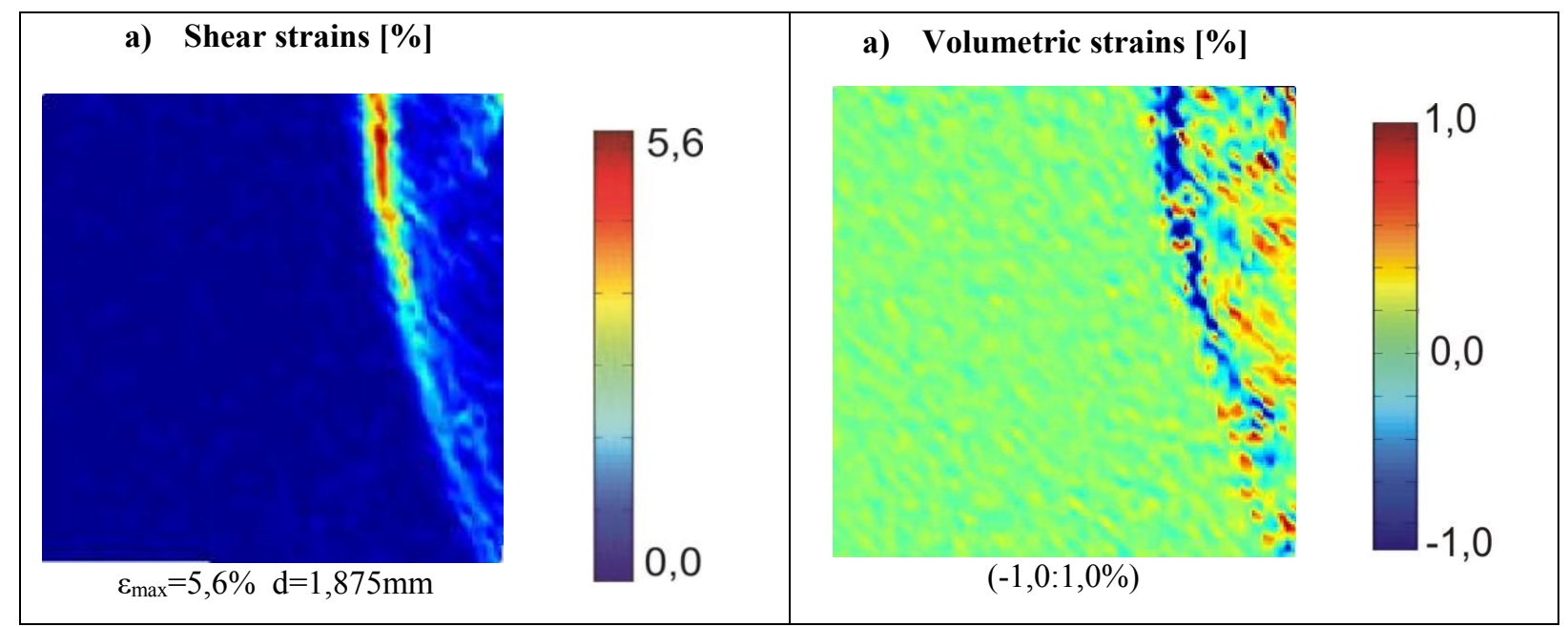

Fig. 8. Third episode of localization: in addition to the main shear band, there are weaker shear bands, dividing the wedge into more rigid segments (compressed - red, Fig.8b). In the course of the main band there are clear areas of compression (red) instead of dilation (blue). 
study, which is obviously related to the granular structure of material. This structure is also responsible for the small dispersed dilatancy, which can be seen in Fig.3b (blue), even though only the macro-sample is compressed on a scale.

However, as can be seen in Fig.3b, its concentration (yellow and red) predominates, so that the average sample reaction can be described according to the assumptions of continuous medium mechanics. Studying analogous deformation fields for other tests in the initial loading phase, it was possible to observe not only a continuous area of dispersed deformation, as in Fig.3, but also clear periodic bands. The observed bands, were dominated by compaction of the medium (yellow and red), which suggests that they could be classified as well known, e.g. from the mechanics of rocks, compaction bands.

Niedostatkiewicz et al. [9] have shown, by comparing the results of 2D PIV and 3D X-ray tomography, that the effect of friction along a transparent wall on measured displacements during planar granular flow is negligible.

\subsection{Deformation cycles}

After reaching the target load level, in the further course of Test 4, wall displacements were given, using their unit increment $\delta \mathrm{d}$, equal to $0.0625 \mathrm{~mm}$. Reaction of material to the horizontal wall displacement at the determined external load was characterized by a clear repeatability. The following phases could be distinguished:

Phase 1: Stage preceding the first localized shear band (Fig.4) - showing distributed shear and volumetric strains in the area forming a pronounced straight wedge (Fig.4a). On the edge of this wedge the dispersed dilatancy prevails (Fig.4b, blue), while inside the wedge the dispersed compression dominates (yellow and red).

Phase 2: 1st localization episode - initial shear band (Fig.5) - deformations concentrate inside a straight shear band, which does not cover the whole height of the model. Fig. 5b shows an increase in volume (dilatant, blue) along the entire localization length, while the compression (red) on the edges - the interior of wedge does not undergo significant deformation (Fig.5a).

Phase 3: 2nd localization episode - the main curvilinear shear band is formed, covering the whole height of model (Fig.6): Deformations of the medium are concentrated inside this band, inside the wedge are visible remains of the original shear band, as shown in Fig.5. The amount of deformation depends on the location inside the main band (the highest values occur in its upper part, near the loaded edge). Volume increase (blue) occurs along the entire localization length, compression (yellow and red) inside the wedge and on the outside of shear band (Fig.6b).

With further deformation progress, the values of shear and volumetric strains inside the shear band equalize, with wall displacement of about $2.5 \mathrm{~mm}$ (approximately two mean grain diameters), shear strains and dilatancy reach their maximum values along the entire location length. This phase completes the development of a single localization of deformations in the area behind the wall. The inside of wedge is evenly compressed at this stage.

Phase 4: Stage after the 2nd localization episode (Fig.7): Evenly distributed deformations can be observed in the area forming the curvilinear wedge, including the inside of area bounded by the shear band in Fig. 6 .

Dilatancy predominates in this area (increase in volume, blue), but along some directions it is also possible to observe compression (red). Phase 4 is analogous to Phase 1, it differs only in the extent of distributed deformation zone (larger wedge, covering the whole height of the model).

Phase 5a: 3rd localization episode (Fig.8): in addition to the main shear band similar to the 1 st and 2 nd localization, there are weaker shear bands, dividing the wedge into more rigid sections (red - compression, Fig.8b).

In the course of main band there are places where compression (red) instead of dilation (blue) appears, which is probably due to the activation of a family of additional weaker localization inside the wedge, which cross the main band at the angle.

Phase 5b: 3rd localization episode (Fig.9): state of advanced deformation inside the shear band approximately uniform distribution of shear strain can be observed (Fig.9a) and alternately occurring compression (red) and dilation inside the shear band (blue, Fig.9b).

Fig. 10 shows in enlarged view the area of shear band in Fig.9 in order to show more precisely the alternating compression and dilation areas inside the shear band. Such a phenomenon has already been noted in the literature $[1,10]$, but it has not been possible to obtain such an accurate image.

In the whole Test 4 we can distinguish 13 cycles, taking place according to the scheme described above. If the cycle starts with e.g. the 2nd localization episode, it is followed by a phase of distributed deformation (as in Phase 4), which lasts for several elementary wall displacements, until the next localization episode.

\section{Conclusions}

The experimental data presented in this paper show that the deformation of granular sample behind retaining wall can undergo some cyclic changes. The nature of the cycles is not clear - it is probably related to some micromechanical features of granular materials, which are recently extensively studied in many research centers in the world.

Such cyclic behavior could presumably explain the cyclic changes of earth thrust on retaining walls, reported in a number of papers $[9,11,12,13]$, but drawing any decisive conclusions needs further research. Employing very precise DIC (PIV) method can help to relate micro and macro-scale behavior of granular materials.

\footnotetext{
* Corresponding author: magdalena.pietrzak@tu.koszalin.pl
} 


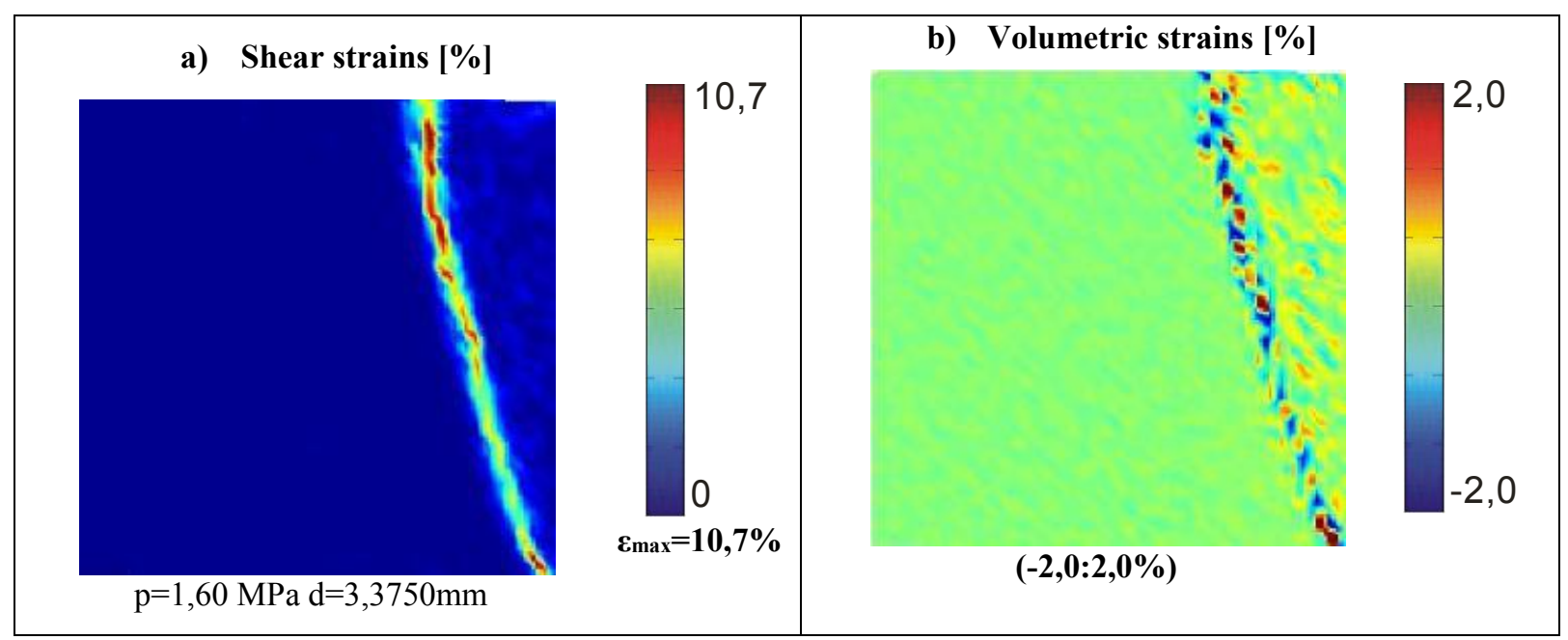

Fig. 9. Advanced deformation: approximately uniform distribution of shear strains (Fig.9a) and alternately occurring compression (red) and dilation (blue, Fig.9b) inside the shear band.

a) Shear strains $[\%]$

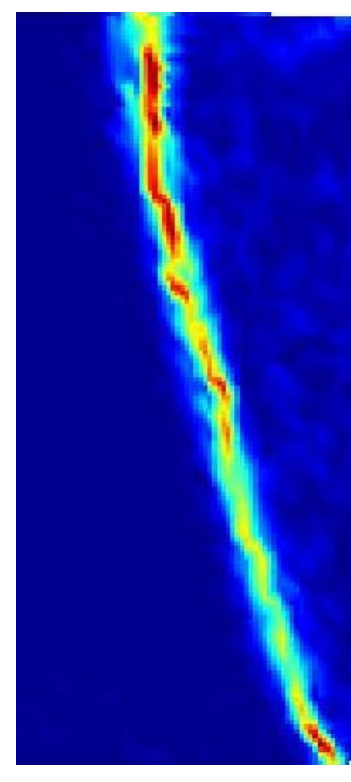

$\varepsilon_{\max }=10,7 \%$ b) Volumetric strains [\%]

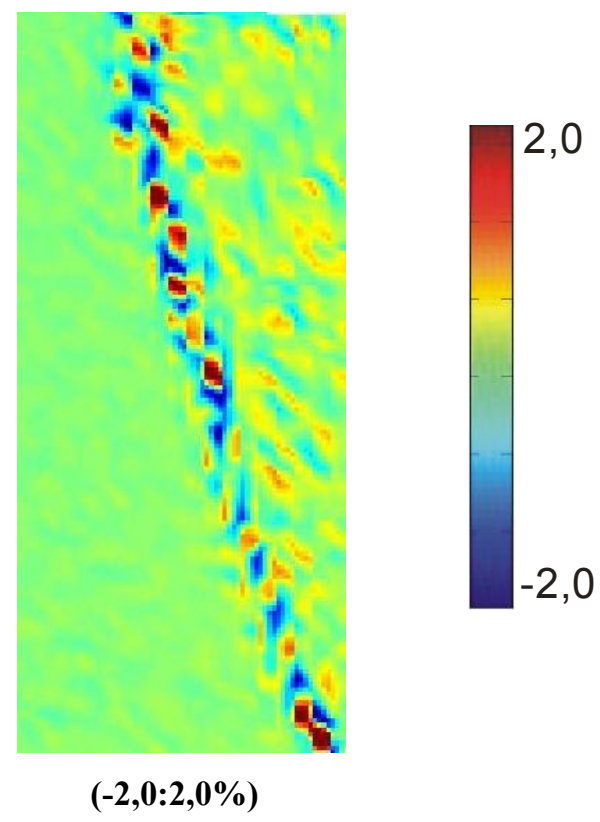

Fig. 10. Test 4: enlarged of deformation localization in the advanced deformation phase: approximately uniform distribution of shear strains (Fig.10a) and alternately occurring compression (red) and dilation (blue, Fig.10b) inside the shear band.

\section{References}

1. D. Lesniewska, D. Muir Wood, J Eng Mech 135:1038-1054 (2009)

2. D. Lesniewska, D. Muir Wood, Geotechnique, 60, doi: 10.1680/geot.2010.60.00.1 (2010)

3. D. Muir Wood, D. Lesniewska, Granul Matt 13:395415 (2011)

4. M. Pietrzak, D. Lesniewska, Studia Geotechnica et Mechanika, XXXIV, 4 : 69-77 (2012)

5. D. Lesniewska, M. Pietrzak, Springer Series in Geomechanics and Geoengineering, 273-278 (2015)

6. D. J White, D. J., W. A. Take, M. D. Bolton, Geotechnique 53, 619-631 (2003)
7. D. J. White, W. A. Take, Technical Report D-SOILSTR322. Cambridge University Engineering Department (2002)

8. D. J White, M. Randolph, B. Thompson, Int. J. Phys. Model. Geotech. 3, 1-12 (2005)

9. M. Niedostatkiewicz D. Leśniewska, J. Tejchman, Strain 47, 218-231(2011)

10. Rechenmacher A.L., Abedi S., Chupin O., Orlando A.D., Acta Geotechnica 6: 205-2017 (2011)

11. D. Leśniewska, Analysis of shear band pattern formation in soil, Polish Academy of Science, Gdańsk Poland (2000)

12. D. Lesniewska, Z. Mroz, Geotechnique 50, No 5, 521-536 (2000)

13. Ł.Widuliński, J.Tejchman, D.Leśniewska, J.Kozicki Int. J. Solids Struct., 48, 1191-1209 (2011)

\footnotetext{
* Corresponding author: magdalena.pietrzak@tu.koszalin.pl
} 